connus par ailleurs. Nous posons des questions, en demandant à une fine dissection anatomique d'y répondre.

Tout ceci nous amène à penser que dans un quartier atteint par l'infection : infection descendante dans le cas de tuberculose non granulique, infection ascendante avec le streptocoque et les autres microbes qui suivent la voie centripète, à côté de lobes ou de lobules qui sont apparemment sains, peut-être même tout à fait sains, il en est d'autres qui sont touchés par le processus inflammatoire. Un lait malade, extrait de ce quartier, doit done être considéré comme un mélange du lait des parties saines et du lait de parties nettement infectées, plus ou moins enflammées.

Ces considérations anatomiques ne nous permettent pas d'aller plus loin; il nous faut passer maintenant sur le terrain de l'histologie.

Là nous trouvons essentiellement deux cellules tout à fait différentes: la cellule du parenchyme propre, cellule noble, spécifique, sécrétrice du lait, regardant par sa face apicale vers la lumière de l'acinus, plongeant par sa base dans le liquide, véritable milieu intérieur - plus que le sang - qui remplit les espaces lacunaires constitués par les aréoles d'une sorte d'éponge dont les travées sont conjonctives.

La réaction inflammatoire est essentiellement conjonctive ; la cellule noble, quand elle est touchée par les produits irritants que certains agents infectieux, mieux que d'autres, élaborent, est troublée dans son fonctionnement normal. Puis, si le processus n'évolue pas vers la guérison, elle se nécrose, et encerclée qu'elle est par une réaction conjonctive plus ou moins visible, finit par disparaître.

(A suivre.)

\title{
LE DOSAGE DE LA MATIÈRE GRASSE DANS LES LAITS ÉCRÉMÉS
}

par

JE.AN PIEN

Ingénieur chimiste (I. C. R.),

Docteur ès Sciences,

Directeur des Laboratoires des

"Fermiers Réunis ». et

\author{
Mile G. MAURICE \\ Chimiste
}

à la Société des « Fermiers Réunis ».

\section{1 er ARTICLE.}

Pour le dosage de la matière grasse dans le lait entier, on dispose de plusieurs méthodes qui donnent des résultats pratiquement identiques et suffisamment exacts. Les écarts constatés entre les différentes méthodes (Röse-Gottlieb, Adam, Gerber, Höyberg) sont faibles, ainsi que plusieurs auteurs l'ont démontré, notamment Ch. Brioux [1] et ORLA-JENSEN [2], et sans importance pratique.

Il n'en est plus du tout de même en ce qui concerne l'analyse des laits écrémés. L'erreur absolue de 0 gr. 5 ou même de 1 gr., en général 
négligeable pour un lait entier titrant 40 gr., présente une importance considérable si elle se reproduit sur des laits écrémés possédant des taux butyreux de l'ordre de 1 ou 2 gr., ou même moins. Le contrôle de la marche des écrémeuses, l'appréciation de la valeur du petit-lait en vue de la fabrication de la caséine, nécessitent la connaissance aussi exacte que possible de la teneur en matière grasse du lait écrémé. Il faudrait, selon nous, que l'erreur ne dépassât point 0 gr. 2 par litre au maximum.

Le but de cette note est l'étude de la valeur respective des méthodes Adam, Röse-Gottlieb et Gerber, eu égard à la question qui nous intéresse.

Le but de notre étude étant précist, il nous faut définir le principe qui a servi de guide à nos recherches :

$1^{\circ}$ Appliquer les méthodes d'extraction à de grands volumes de lait ; analyser l'extrait obtenu (dosage des impuretés) ;

$2^{\circ}$ Le chiffre de la matière grasse pure sera calculé en défalquant de l'extrait précéder t les impuretés dosées ;

30 Appliquer la méthode Gerber dans les conditions optima, à l'aide d'un butyromètre spécial que nous avons créé.

$4^{\circ}$ Comparer les chiffres de la matière grasse obtenus par les trois méthodes et expliquer les différences, s'il y a lieu.

D'où le plan de l'étude :

Première partie. - Etude des méthodes par épuisement à l'éther (Techriques suivies, Résultats obtenus).

Deuxième partie. - Etude de la móthode Gerber (Technique suivie, Résultats obtenus).

Troisième partie. - Résumé et Conclusions.

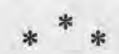

PREMIERE PARTIE.

ÉTUDE DES MÉTHODES PAR ÉPUISEMENT A L'ÉTHER.

I.

TECHNIQUES SUIVIES.

\section{TECHNIQUE DE LA MÉTHODE D'ADAM.}

Les avis sont assez partagés dans les divers laboratoires qui utilisent la méthode Adam sur le mode opératoire précis à appliquer. Le mieux était de recourir aux sources. Un mot d'historique ne paraît donc pas superflu. Dans sa thèse [3] et dans les études publiées à la même époque [4] [5], ADAM s'est proposé (en 1879) la critique et l'amélioration du procédé Marchand [6], qui datait lui-même de 1854.

MARCHAND, à l'origine, traitait le lait par de la soude et opérait 
des épuisements successifs à l'éther. Un peu plus tard, ce même auteur perfectionnait sa méthode par l'usage d'alcool à $86^{\circ}-90^{\circ}$ (qui facilitait la séparation de la couche éthéro-butyreuse) et proposait son lactobutyromètre. ADAM a surtout étudié systématiquement le rôle de l'alcool et de son titre; il a démontré et expliqué l'erreur commise par MaRCHAND (qui, d'ailleurs, faisait appel à un terme correctif constant pour l'expression des résultats) : l'alcool à 860-90 est trop concentré; le titre optimum est $75^{\circ}$, fixé et adopté par ADAM.

La méthode initiale d'Adam comporte dono l'addition à $10 \mathrm{~cm}^{3}$ de lait de :

1 goutte de soude, puis $10 \mathrm{~cm}^{8} \mathrm{~d}^{\prime}$ alcool à $75^{\circ}$, puis $11 \mathrm{~cm}^{3}$ d'éther à $65^{\circ}$.

ADAM préconise également le rinçage à l'éther de son appareil. En opérant ainsi, cet auteur observe que l'extrait obtenu contient environ $1 \mathrm{cgr}$. de matières caséeuses dont on peut se débarrasser presque complètement en reprenant ce résidu par l'éther.

Magnier de la Source [7] démontre, la même année (1879), que la nouvelle méthode ainsi appliquée donne des résultats suffisamment exacts : la couche hydro-alcoolique inférieure ne renferme aucun corps gras ; quant à la couche éthérée supérieure, en la relavant par le réactif d'Adam, on obtient un chiffre un peu plus faible (de 0,3 à 1 gr. par litre de lait), que MAGNIER DE LA SouRCE attribue à un peu de caséine contenue dans la couche éthérée de l'essai direct.

En 1881, AdAM améliore sa méthode [8]. Il modifie la forme de son galactotimètre. Il modifie son réactif en remplaçant la soude par l'ammoniaque et il propose la formule suivante :

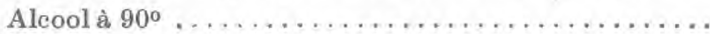

$833 \mathrm{~cm}^{3}$

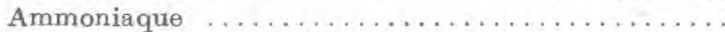

$30 \mathrm{~cm}^{3}$

Eau q.s. p.

$1.000 \mathrm{~cm}^{3}$

que l'on complète ainsi :

$\begin{array}{ll}\text { Alrool a mmoniacal à } 75^{\circ} \text {, ci-dessus } \ldots \ldots \ldots \ldots \ldots \ldots \ldots \ldots \ldots \ldots \ldots \ldots \ldots \ldots \ldots \ldots \ldots \ldots \ldots \ldots \ldots \ldots \ldots \ldots & 100 \mathrm{~cm}^{3} \\ \text { Ether pur à } 65^{\circ} \ldots \ldots \ldots \ldots \ldots \mathrm{cm}^{3}\end{array}$

ADAM modifie aussi son mode opératoire.

Après avoir décanté la couche infériọure, il lave la couche éthérée en versant dans l'appareil (contre las parois) de l'eau distillée jusqu'à parfaire lo volume de $32 \mathrm{~cm}^{3}$, laisse reposer, décante à nouveau, recueille à part la liqueur éthérée et rince finalement l'appareil à l'éther.

D'après ADAM, ce lavage à l'eau de la couche éthérée, augmenterait l'exactitude de la méthode, qui atteindrait désormais 0,1 à 0,2 par litre de lait.

Cette méthode est encore appliquée de nos jours, exactement comme ADAM l'a décrite en 1881, par de nombreux laboratoires, et, en particulier, par le Laboratoire municipal de Paris [9].

Remarque. - On sait qu'AdAM a également proposé une méthode 
volumétrique, dans laquelle on procède d'abord comme dans la méthode gravimétrique précédente, pour, ensuite, évaporer l'éther dans l'appareil lui-même et lire le volume de matière grasse pure. ADAM préconise, pour mieux rassembler le beurre, d'effectuer le. lavage avec de l'acide acétique à $15 \%$.

Hinard [10] recommande de faire ee lavage à l'acide acétique, même dans le cas du dosage gravimétrique d'Adam (après un premier lavage à l'eau). Ce lavage acide enlèverait 2 à $3 \mathrm{mgr}$. d'impuretés (soit 0,2 à 0,3 par litre), ce qui augmenterait la précision de l'analyse. Cependant le Laboratoire municipal de Paris croit cette prescription en général inutile ([9], page 66).

De quelle base allions-nous donc partir pour soumettre la méthode d'Adam à une étude critique pour l'analyse des laits écrémés? Nous ne pouvions mieux faire que de nous borner aux indications même de l'auteur, données, en 1881, après perfectionnement de sa méthode, indications qui sont reproduites très fidèlement dans l'ouvrage de KLING [9] et suivies au Laboratoire municipal de Paris. Mais nous ne pouvions pas nous désintéresser de la remarque de Hinard au sujet du lavage acétique pour l'analyse en poids, et nous avons également effectué des essais sur ce procédé.

Les modes opératoires exactement suivis par nous sont done les suivants :

\section{MÉTHODE D'ADAM CLASSIQUE.}

a) Réactif, - On prépare le réactif suivant (1) :

\begin{tabular}{|c|c|c|}
\hline Alcool à $90^{\circ}$ & $333 \mathrm{em}^{3}$ ou & $833 \mathrm{~cm}^{3}$ \\
\hline Eau distillée & $55 \mathrm{~cm}^{3}$ ou & $137 \mathrm{~cm}^{3}$ \\
\hline Ammoniaque à $22^{\circ}$. & $12 \mathrm{~cm}^{3}$ ou & $30 \mathrm{~cm}^{3}$ \\
\hline Cther à $65^{\circ} \quad \ldots$ & $440 \mathrm{~cm}^{3}$ ou & $.100 \mathrm{~cm}^{3}$ \\
\hline
\end{tabular}

b) Extraction. - On introduit dans le galactotimètre d'Adam $10 \mathrm{~cm}^{3}$ de lait et $22 \mathrm{~cm}^{3}$ du réactif précédent (que l'on verse lentement pour éviter de former un caillot volumineux).

On bouche l'appareil avec un bauchon de liège taillé en biseau.

On retourne plusieurs fois l'appareil pour bien mélanger les liquides, puis on agite assez violemment pendant une minute.

On obtient un liquide translucide, sans aucun flocon.

On laisse reposer une demi-heure.

c) Soutirages. - On soutire la couche inférieure jusqu'à ce qu'il n'en reste dans l'appareil que $\mathbf{l} \mathrm{cm}^{3}$ environ. On rebouche l'appareil, puis, en le tenant obliquement, on le roule entre les doigts pour faire descendre les gouttelettes de liqueur aqueuse adhérentes aux parois.

On laisse reposer encore cinq minutes.

On soutire à nouveau jusqu'à ce que la liqueur éthérée atteigne exactement le robinet, sans toutefois le dépasser.

(1) Cette formule est exactement la même que celle proposée par ADAM en 1881 (voir plus haut). 
d) Lavage de la couche éthérée. - On verse de l'eau distillée dans lè galactotimètre en la faisant couler doucement contre les parois pour ramener lo volume total à $32 \mathrm{~cm}^{3}$. On n'agite pas la couche éthérée avec l'eau. Cette opération n'a pas tant pour but de laver l'éther, que de rincer les parois auxquelles adhère encore un peu de liqueur hydroalcoolique contenant des matières étrangères en solution.

On laisse reposer vingt minutes. On décante cette eau de lavage jusqu' $1 \mathrm{~cm}^{3}$. On roule encore l'appareil entre les doigts, on laisse reposer encore cinq minutes et on soutire avec précaution ce qui reste d'eau jusqu'à ce que le niveau de la couche éthérée dépasse le robinet et atteigne la pointe extrême de l'appareil.

e) Evacuation de la couche éthérée et rinçage de l'appareil. On reçoit alors la couche éthérée dans une eapsule de verre tarée.

On rince le bouchon de liège et lappareil avec quelques centimètres eubes d'éther sulfurique que l'on joint au contenu de la oapsule.

Finalement, on évapore l'éther, on sèche la capsule à $98^{\circ}-100^{\circ}$ pendant trente. minutes au moins, on refroidit à l'exsiccateur et on pèse.

2 'MODE OPERATOIRE DE HINARD [10].

Il ne diffère du précédent que par le point suivant :

Quand la couche éthérée a été lavée à l'eau (paragraphe $d$ ci-dessus), on pro. cède à un lavage analogue avec de l'acide acétique à $15 \%$.

Les autres opérations sont effectuées comme dans le mode opératoire d'Adam.

\section{TECHNIQUE DE LA MÉTHODE RÖSE-GOTTLIEB.}

BRUno Röse [11] propose, en 1888, une méthode basée sur le même principe général que la méthode d'Adam, mais qui en diffère, néanmoins, par les points suivants :

$1^{0} \mathrm{~L}$ 'ammoniaque est introduite directement dans le lait et non dans le réactif .

$2^{\circ}$ L'éther sulfurique employé est additionné d'éther de pétrole, dont la présence aurait pour but d'insolubiliser les impuretés passées dans l'éther sulfurique. (Gotтlies même va plus loin et n'utilise que de l'éther de pétrole pur pour l'extraction de la matière grasse.)

Notons en passant que les travaux de MeILLÈre [12] [13] [14] sur la méthode d'Adam, l'ont amené indirectement à confirmer certaines des indications de BRUno RösE : alcalinisation directe du lait, usage d'éther de pétrole. Mais le procédé Meillère se rattache à l'Adam par l'usage d'alcool à $75^{\circ}$, alors que la méthode RöseGottlieb s'en écarte par l'usage d'alcool beaucoup plus concentré.

La présence d'éther de pétrole et la très forte alcalinisation du lait permettent d'éviter les inconvénients signalés par ADAM, dus à l'usage d'alcool de haut titre dans la méthode Marchand. Le butyromètre utilisé dans cette méthode (tube Roehrig) a été très heureusement modifié par BRIoux [15], à qui nous empruntons le mode opératoire suivant :

10 Introduire dans le butyromètre, à l'aide d'une pipette graduée, $10 \mathrm{~cm}^{3} \mathrm{de}$ lait, puis $1 \mathrm{em}^{3}$ d'ammoniaque ordinaire; agiter pendant une minute environ, pour bien solubiliser la caséine. 
$2^{\circ}$ Ajouter $10 \mathrm{~cm}^{3}$ d'alcool à $95^{\circ}$ et agiter de nouveau en amenant lè mélange dans l'ampoule centrale, mais sans renverser complètement le butyromètre.

$3^{\circ}$ Ajouter $25 \mathrm{~cm}^{3}$ d'éther ordinaire préalablement saturé d'eau ; boucher l'appareil, en ayant soin que les sillons du bouchon rodé ne correspondent pas aux ouvertures du-tube, et agiter fortement le mélange en renversant plusieurs fois l'appareil.

$4^{\circ}$ Ajouter $25 \mathrm{~cm}^{3}$ d'éther de pétrole de densité voisine de 0,65 et agiter de nouveau énergiquement.

Le butyromètre, placé verticalement sur un support approprié, est abandonné pendant une heure ou deux, puis on détermine, par deux lectures, le volume total de la solution éthéro-alcoolique.

On prélève ensuite une partie aliquote de cette liqueur éthérée, on évapore, sèche, et pèse ; on en déduit la matière grasse totale extraite.

Dans tous nos essais sur la méthode Röse-Gottlieb, nous avons utilisé le butyromètre Brioux.

\section{TECHNIQUE DE L'ANAL YSE DES RÉSIDUS D'EXTRACTION.}

Les nombreuses extractions que nous avons réalisées (50 pour chaque méthode) ont été rassemblées dans quatre capsules de verre seulement (correspondant chacune à $125 \mathrm{~cm}^{3}$ de lait), de manière à nous éviter, en vue de l'analyse, des reprises pénibles et difficiles des résidus, ce qui eût été une source de pertes et d'erreurs. Ainsi, certaines capsules ont pu être réservées chacune au dosage d'un seul élément.

Nous avons dosé dans nos résidus :

Le lactose et les matières protéiques sur le contenu de deux capsules réunies (correspondant à $250 \mathrm{~cm}^{3}$ de lait);

Les chlorures sur une seule capsule (correspondant à $125 \mathrm{~cm}^{3}$ de lait);

Les lécithines sur une capsule (correspondant à $125 \mathrm{~cm}^{3}$ de lait).

Voici les modes opératoires que nous avons fixés :

10 Mise en solution. - Les capsules sont d'abord lavées à l'éther seul, puis à l'eau bouillante. Les solutions aqueuse et éthérée obtenues sont rassemblées dans un unique ballon de $100 \mathrm{~cm}^{3}$. On porte ce ballon au bain-marie à $60^{\circ}$ pour chasser l'éther. On a finalement une liqueur contenant une suspension de matière grasse et une solution des sucres et autres matières solubles dans l'eau. On refroidit et on complète à $100 \mathrm{~cm}^{3}$. (Les capsules lavées sont conservées pour l'étude des matières insolubles.)

$2^{\circ}$ Dosage du lactose. - On traite deux capsules, dont on réunit l'extrait aqueux dans un seul ballon de $100 \mathrm{~cm}^{3}$. On filtre cette solution. Sur $40 \mathrm{~cm}^{3}$ (ramenés à 20 par concentration et correspondant à $100 \mathrm{~cm}^{3}$ de lait), on dose le lactose par la méthode de G. Bertrand.

$3^{\circ}$ Dosage des matières protéiques. - Les capsules qu'on a lavées à l'éther et à l'eau sont maintenant lavées à l'acide sulfurique 
pur pour dissoudre (s'il y a lieu) les matières protéiques qu'elles peuvent encore contenir. La liqueur obtenue est transvasée dans un ballon Kjeldahl.

Le filtrat du dosage des sucres y est également introduit, ainsi que le reste de la solution aqueuse du ballon de $100 \mathrm{~cm}^{3}$, le filtre et les eaux de rinçage de tous ces récipients. On a ainsi rassemblé la totalité des extraits de deux capsules correspondant à $250 \mathrm{~cm}^{3}$ de lait.

On procède à une attaque Kjeldahl après concentration de tous ces liquides, et on termine le dosage de l'azote par les procédés habituels.

$4^{\circ}$ Dosage des chlorures. - La liqueur provenant du traitement d'une capsule (correspondant à $125 \mathrm{~cm}^{3}$ de lait) par l'éther, par l'eau bouillante, puis par l'acide nitrique dilué $(10 \%)$ chaud, est filtrée, additionnée de $5 \mathrm{~cm}^{3}$ de $\mathrm{NO}^{3} \mathrm{Ag} \mathrm{N} / 10$; puis après dix minutes de contact entrecoupées d'agitations, on titre en retour au sulfocyanure N/10 en présence d'alun ferrique.

$5^{\circ}$ Dosage de la chaux. - Une partie de la solution aqueuse précédente a été réservée au dosage de la chaux : on alcalinise par l'ammoniaque, rend acide par l'acide acétique et précipite par l'oxalate d'ammoniaque à chaud.

$6^{\circ}$ Dosage de la lécithine. - On a fait appel à la méthode Bordas-de Raczkowski [16], qui dose l'acide glycérophosphorique de cet élément.

Voici comment nous l'avons adaptée à notre cas particulier :

Le contenu d'une capsule est repris par l'éther sulfurique, qui dissout graisses et lécithines. On transvase dans un ballon pouvant s'atteler à un réfrigérant æ̀ reflux. On évapore le solvant. On traite pendant une heure à l'ébullition (à reflux) par $10 \mathrm{~cm}^{3}$ de $\mathrm{KOH}$ alcoolique environ normale et $10 \mathrm{~cm}^{3}$ d'alcool à $95^{\circ}$. On chasse la majeure partie de l'alcool et rend, acide par $\mathrm{NO}^{3} \mathrm{H}$. Les acides gras sont libérés. La totalitá est reprise plusieurs fois par l'eau bouillante. On soutire à chaque fois dans une ampoule à brome la -partie aqueuse qui contient les glycérophosphates et phosphates.

On filtre, on concentre à l'ébullition, puis à sec au bain-marie dans un bécher.

On oxyde ensuite selon la méthode de Marie [17], en ajoutant sur le résidu sec, $10 \mathrm{em}^{3}$ d'acide nitrique concentré ét en projetant du $\mathrm{MnO}^{4} \mathrm{~K}$ pulvérulent jusqu'à eoloration rose persistante. L'excès de $\mathrm{MnO}^{\prime} \mathrm{K}$ est détruit par un peu de nitrite de soude (à $10 \%$ ) et les vapeurs nitreuses chassées à l'ébullition. On précipite enfin l'acide phosphorique par le molybdate d'ammoniaque et on termine le dosage dans les conditions habituelles.

Le poids de $\mathrm{P}^{2} \mathrm{O}^{5}$ trouvé est multiplié par 1,549 pour avoir l'acide phosphoglycérique ou par 11,16 pour avoir la lécithine correspondante.

Nota. - Il eût été plus simple de séparer la lécithine pure par l'action de solvants différentiels en suivant la technique de GLIKIN [18]. La trop faible quantité de substance mise en cuvre nous y a fait renoncer. 
II.

RÉSULTATS OBTENUS.

1. poids des extraits. - Tous nos essais ont été faits sur le même lait écrémé contenant encore 2 à $3 \mathrm{gr}$. de matière grasse : le premier jour sur le lait frais, les trois jours suivants sur le même lait bichromaté.

Chaque jour, 12 ou 14 dosages étaient effectués par chacune des méthodes.

1 . Moyennes des résultats de chaque journée.

\begin{tabular}{|c|c|c|c|c|}
\hline \multirow{2}{*}{ a } & \multirow{2}{*}{$\frac{\text { Lait frais }}{1^{\mathrm{er}} \text { jour }}$} & \multicolumn{3}{|c|}{ Lait bichromaté } \\
\hline & & $2^{e}$ jour & $3^{\text {e }}$ jour & $4^{e}$ jour \\
\hline Méthode d'Adam & 2,72 & 2,76 & 2,81 & 2,78 \\
\hline Mode opératoire Hinard & 2,58 & 2,75 & 2,78 & 2,74 \\
\hline Méthode Röse-Gottlieb & 2,70 & 2,78 & 2,79 & 2,68 \\
\hline
\end{tabular}

Si l'on excepte le chiffre obtenu sur lait frais par le mode opératoire Hinard, on peut en conclure que les trois méthodes donnent des résultats identiques. On ne constate que de faibles variations entre les dosages des différentes journées.

Il est donc intéressant de montrer les chiffres obtenus à la fin de la quatrième journée (ramenés à 1 litre de lait) sur les diverses capsules qui ont servi ; car celles-ci ont marché en parallèle d'un bout à l'autre de l'expérience et renferment, toutes, des extraits de laits des quatre jours.

Méthode Adam (50 dosages):

$$
\left.\begin{array}{l}
2,77 \\
2,78 \\
2,75 \\
2,77
\end{array}\right\} \text { Moyenne générale; } 2,77
$$

Méthode Röse-Gottlieb (50 dosages) : 2,73

$$
\left.\begin{array}{l}
2,73 \\
2,74 \\
2,75
\end{array}\right\} \text { Moyenne générale; } 2,74
$$

Mode opératoire de Hinard (12 dosages) :

$$
\text { 2,71\} Moyenne générale ; 2,71. }
$$

\begin{tabular}{|c|c|c|}
\hline Méthode d'Adam : & Dosage minimum & $\ldots$ \\
\hline & Dosage maximum & $\ldots \ldots \ldots$ \\
\hline & Ecart maximum & \\
\hline Méthode Röse-Gottlieb & Dosage minimum & $\ldots \ldots \ldots \ldots$ \\
\hline & Dosage maximum & $\ldots \ldots \ldots$ \\
\hline & Ecart maximum & $\ldots \ldots$ \\
\hline
\end{tabular}

20 Ecarts. - Pour pouvoir attacher une certaine valeur à ces résultats, il faut connaître l'amplitude de nos écarts : 
Ces écarts nous montrent que la méthode d'Adam est un peu plus régulière que la méthode Gottlieb. La raison en est sans doute dans ce que, dans cette dernière, on ne prélève, à chaque essai, qu'une partie aliquote du volume total de la couche éthérée. Il y aurait lieu de faire intervenir aussi la variation de composition du lait entre le premier et le quatrième jour (1).

Une nouvelle série de dosages effectués huit jours plus tard sur le même lait conservé dans une pièce chaude nous a donné 2,70 à l'Adam et 2,74 au Gottlieb.

$3^{\circ}$ Conclusions. - Les méthodes d'Adam et de Röse-Gottlieb ont donné, sur le lait écrémé qui nous a servi, des résultats identiques et pratiquement constants.

Il nous reste à voir si ces chiffres correspondent à de la matière grasse pure ou si ces deux méthodes sont identiquement fausses.

2. ANALYSE des extraits. - On a vu que les différentes capsules (9 au total) contiennent des extraits identiques. On pouvait done indifféremment prendre l'une ou l'autre (dans chaque méthode) pour effectuer les dosages de matières étrangères suivant les techniques indiquées.

Voici les résultats obtenus, ramenés à un litre de lait :

\begin{tabular}{|c|c|c|}
\hline 1 & $\begin{array}{l}\text { Méthode } \\
\text { d'Adam }\end{array}$ & $\begin{array}{c}\text { Méthode } \\
\text { Röse- } \\
\text { Gottlieb }\end{array}$ \\
\hline 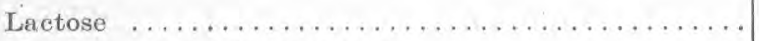 & 0,028 & 0,015 \\
\hline 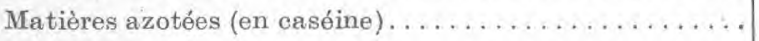 & 0,070 & 0,075 \\
\hline 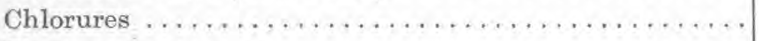 & 0,006 & 0,006 \\
\hline 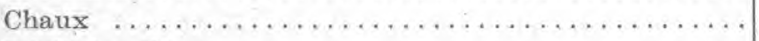 & traces & traces \\
\hline 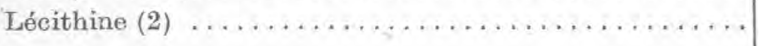 & 0,222 & 0,214 \\
\hline $\begin{array}{l}\text { Total des matières étrangères dosées } \ldots \ldots \ldots \ldots \ldots \ldots \ldots \\
\text { Or, on a eu : }\end{array}$ & 0,326 & 0,310 \\
\hline $\begin{array}{l}\text { Extrait total par litre } \ldots \ldots \ldots \ldots \ldots \ldots \ldots \ldots \ldots \ldots \ldots \\
\text { D'où : }\end{array}$ & 2,77 & 2,74 \\
\hline Matière grasse supposée pure $(3) \ldots \ldots \ldots \ldots \ldots \ldots$ & 2,444 & 2,430 \\
\hline
\end{tabular}

(1) On peut se demander pourquoi nous n'avons pas opéré d'un coup sur $500 \mathrm{~cm}^{3}$ de lait en appliquant chaque méthode avec les quantités voulues de réactifs ; c'eût été plus simple que d'effectuer 112 dosages sur $10 \mathrm{~cm}^{3}$. En fait, nous n'avions pas le droit d'opérer ainsi. Il n'est pas possible; sur $500 \mathrm{~cm}^{3}$ de lait, d'appliquer exactement le mode opératoire fixé pour des dosages normaux sur $10 \mathrm{~cm}^{3}$. La forme des appareils (qui a son importance), le mode d'agitation, l'influence des soutirages, le lavage de la couche éthérée dans la méthode d'Adam, n'eussent pas été respectés, et un dosage sur 1/2 litre de lait n'est pas du tout com. parable à la somme de cinquante dosages successifs effectués avec soin. Nous avons d'ailleurs exécuté, à titre d'essais préliminaires, ces dosages sur de grands volumes de lait dans des 
Remarque. - Des dosages de lécithine sur le lait lui-même nous ont donné des chiffres voisins de 0,2 par litre. Il semblerait done que toute la lécithine du lait soit passée dans l'extrait.

III.

CONCLUSTONS.

La matière grasse extraite dans la méthode d'Adam ou dans la méthode Gottlieb contient deux sortes d'impuretés :

$1^{\circ}$ Des matières non grasses (lactose, caséine, sels...), en très petite quantité (ici, environ 0 gr. I pour un litre de lait);

$2^{\circ}$ Une quantité de lécithine un peu plus importante $(0$ gr. 2 pour un litre de lait) (1), quantité qui semble correspondre à la lécithine même du lait. Cette lécithine représente ici environ $8 \%$ du poids de l'extrait total des deux méthodes.

Chapman [19], qui a fait également des dosages de lécithine sur les extraits obtenus par la méthode Röse-Gottlieb dans le cas de laits écrémés, trouve que ces extraits en contiennent environ $10 \%$. Dans le cas où les doses de lécithines qu'on peut ainsi trouver seraient proportionnelles à la teneur en graisse pure de l'extrait éthéré obtenu (ce qui est possible, puisque la lécithine accompagne la matière grasse), on pourrait voir, dans nos résultats, une confirmation de ceux de Chapman. En tout cas, nous pouvons affirmer que, si on considère la lécithine comme devant faire partie de la matière grasse normale du lait, les méthodes Adam et Gottlieb-Röse nous ont donné des résultats parfaits (matière grasse pure à 0 gr. 1 près par litre) et identiques.

(Suite des notes de la page 277).

récipients de plusieurs litres. Ils ne nous ont pas donné satísfaction. Ia ma tière grasse cbtenue était beaucoup plus souillée que celle de nos dosages normaux, ainsi qu'on s'en rendra compte ci-après (analyse des extraits obtenus).

(2) On pourra nous objecter qu'il n'y avait pas lieu de compter la lécithine corme une matière étrangère, puisqu'on la considère comme un éther de la glycérine dont deux fonctions alcool sont liées à des acides gras, la troisième étant liée à la choline par l'intermédiaire de l'acide phosphorique. La lécithine peut, en effet, être considérée comme une graisse. Mais nous aurons ci-après l'occasion de montrer tout l'intérêt qui s'attache à cette distinction en étudiant la méthode Gerber appliquée aux laits écrémés.

(3) Il faut remarquer que d'autres matières étrangères peuvent encore exister dans l'extrait obtenu en dehors de celles que nous avons dosées. Notre chiffre de 2,43-2,44 repré. sente un maximum de matière grasse et est peut être encore trop élevé. Nous pensons, néan. moins, avoir dosé la majeure partie des impuretés.

(1) On pourrait nous reprocher d'avoir comptê en lécithine, de l'acide phosphorique d'origine minérale. Il n'en est rien ; car, d'une part, l'acide phosphorique minéral aurait amené avec lui une quantité de chaux non négligeable, ce qui n'est pas le cas ; d'autre part, pour le aosage de la lécithine, nous avons repris nos extraits séchés par de l'éther seul, sans eau, ni acide, laissant ainsi insolubilisées dans la capsule les faibles quantités de sels minéraux qu'elles renfermaient. Le phosphore organique de la caséine n'est pas davantage en cause. Il n'y en aurait, dans notre extrait, que 0,001 pour un litre de lait. 


\section{REMARQUE.}

De la nécessité de suivre exactement la technique d'Adam. - A titre d'expérience, nous avons fait un dosage sur un grand volume de lait écrémé (un litre d'un coup) dans un très grand flacon, en négligeant volontairement la technique des soutirages fractionnés et du lavage à l'eau de la couche éthérée. Nous avons pu ainsi apprécier l'erreur commise. Nous avons trouvé un extrait total de 2 gr. 669 , alors que par la méthode correctement exécutée sur $10 \mathrm{~cm}^{3}$, nous trouvions 1,7 (1). Cette surcharge de près de $1 \mathrm{gr}$. contenait $0 \mathrm{gr}$. 480 de lactose et 0 gr. 206 de matières protéiques. Nous n'avons pas dosé les lécithines.

D'autres essais, effectués sur de grands volumes de lait, en négligeant les mêmes précautions, mais en faisant toutefois usage du galactotimètre (ce qui correspondait à des dosages classiques sans lavage et mal soutirés), nous donnaient une masse d'impuretés totales variable, mais contenant presque toujours 40 à $50 \%$ de lactose et 20 à $30 \%$ de caséine.

Tous ees essais montrent l'absolue nécessité de suivre à la lettre le mode opératoire d'Adam décrit plus haut ; ils expliquent les erreurs commises quand on ne se soumet pas à ces prescriptions et donnent probablement la raison des critiques, injustifiées selon nous, dont la méthode d'Adam a été l'objet.

DEUXIEME PARTIE.

\section{ÉTUDE DE LA METHODE GERBER.}

Trois considérations nous ont amenés à envisager l'étudè de la méthode Gerber pour le lait écrémé :

$1^{0}$ Les méthodes d'extraction précédentes exigent un matériel, des réactifs appropriés et surtout beaucoup de soin. Si les preseriptions rappelées ci-dessus ne sont pas appliquées à la lettre, elles donnent des résultats inexacts. Ce sont des méthodes de choix, excellentes entre des mains exercées et ne pouvant pas sortir du laboratoire. Il était donc bon d'étudier pour les laits écrémés la méthode industrielle de Gerber, déjà si intéressante pour les laits entiers.

$2^{\circ}$ Des essais préliminaires nous ont montré que la méthode Gerber ne dose pas les lécithines du lait. Si une distinetion doit être faite entre la matière grasse pure et les lécithines, il est intéressant d'étudier en détail une méthode qui ne les dose pas.

$3^{\circ}$ Enfin, nous avons pu constater qu'en utilisant un butyromètre spécial, mis au point par nous, et une technique appropriée, on pouvait arriver, en gardant le principe de la méthode Gerber, à une grande précision et une grande régularité dans l'analyse des laits écrémés.

Ces trois raisons justifient l'étude à laquelle nous avons procédé.

(1) Il s'agit, bien entendu, d'un lait différent de celui qui a servi aux essais précédents. 


\section{$* *^{*} *$}

I.

TECHNIQUE SUIVIE.

La méthode Gerber, telle qu'on la pratique sur des laits entiers, peut permettre un examen sommaire des laits écrémés.

Mais si on fait usage du butyromètre à lait entier (dont une division de la graduation représente $1 \mathrm{gr}$. de matière grasse par litre ou par kilo de lait, suivant le modèle courant employé), la précision du procédé est extrêmement faible.

L'erreur normale du procédé Gerber classique est de l'ordre de 0 gr. 5, ou moins, ainsi qu'il résulte de nombreux essais exécutés dans nos laboratoires. Si on a soin, en effet, de faire avec le même lait une cinquantaine de dosages sur cinquante tubes différents (pour un seul opérateur) ou sur quinze tubes avec trois opérateurs différents, on s'aperçoit qu'on a 20 à $30 \%$ d'éearts de $\pm 0,5$ autour de la moyenne, $30 \%$ d'écarts inférieurs à cette quantité et 40 à $50 \%$ de eoncordances absolues.

Dans près de la moitié des cas, on ne peut done pas affirmer la précision du demi-gramme et il faut admettre qu'on a quelquefois, dans la pratique courante, des erreurs qui atteignent $1 \mathrm{gr}$. Le butyromètre à lait entier ne peut, pour les laits écrémés, que permettre un triage grossier.

Que valent les butyromètres spéciaux créés pour le dosage des laits écrémẻs ?

Ces appareils sont de plusieurs types différents :

$1^{\circ}$ Les uns sont constitués par un réservoir deux fois plus grand que le butyromètre à lait entier, mais avec une échelle à large diamètre comme ces derniers. Ils n'apportent qu'une sensibilité deux fois plus grande, sans augmentation de la précision absolue.

$2^{\circ}$ D'autres sont constitués par un réservoir de butyromètre à lait entier, avec une échelle légèrement rétrécie. Ils apportent une petite amélioration sur la précision, mais n'augmentent pas la sensibilité.

$3^{\circ}$ D'autres comportent un réservoir ordinaire (comme pour le lait entier), avec une échelle assez fine, terminée par un tube ouvert, sur lequel se visse un petit bouchon. L'usage a depuis longtemps montré que ce type d'appareils est pratiquement inemployable en raison des giclures de matière grasse qui se produisent quand on desserre légèrement le petit bouchon pour permettre la montée de la matière grasse dáns l'échelle.

$4^{\circ}$ Enfin, d'autres (type Siegfeld) comportent un réservoir double, avec une échelle à section rectangulaire extra plate. Outre que cet appareil est très fragile, il offre l'inconvénient suivant : dans la centrifugation d'un lait traité par l'acide Gerber (en présence d'alcool 
amylique), il reste presque toujours des traces d'insoluble (1) qui viennent se placer à la zone de séparation de la couche de matière grasse ; dans les butyromètres ordinaires, ce léger insoluble se déplace avec la zone de séparation ; dans le Siegfeld, il vient tapisser les parois de la fine échelle plate et modifie le volume réel de la matière grasse.

En outre, la partie d'insoluble qui reste à la zone de séparation n'affecte plus la forme d'un disque, mais celle d'un ruban plus ou moins long et déchiqueté, qui est imprégné de matière grasse et ne permet pas toujours d'apprécier aisément le niveau inférieur de la colonne grasse. Il nous est maintes fois arrivé, même, de voir cet insoluble envahir complètement la colonne de matière grasse fondue quand le volume de celle-ci est très faible.

Enfin, le nettoyage de ce tube est une opération irréalisable, par suite de l'impossibilité d'introduire dans le canal l'aiguille creuse d'un laveur Richmond.

L'idéal serait done de faire appel à un appareil ayant les avantages des précédents sans en avoir les inconvénients.

Les conditions à réaliser sont les suivantes :

$1^{\circ}$ Réservoir double (pour augmenter la sensibilité).

$2^{\circ}$ Colonne graduée fine (pour augmenter la précision).

$3^{\circ}$ Colonne graduée à section circulaire pour éviter les inconvénients du tube Siegfeld (obturation partielle par l'insoluble, impossibilité de nettoyage à l'aide d'un laveur Richmond).

$4^{\circ}$ Colonne graduée renforcée pour augmenter la solidité de l'appareil.

$5^{\circ}$ Suppression du bouchon à vis de certains butyromètres à laits écrémés.

1. CREATION D'UN BUTYRométre nOUVEAu POUR LAIT ÉCRÉmÉ. - Nous avons créé un butyromètre répondant à ces.conditions et qui nous donne entière satisfaction (2).

Il a l'aspect d'un butyromètre Gerber classique à réservoir double. L'échelle est gravée sur une tige à section carrée, épaisse. Le canal est à section circulaire, permettant aisément le lavage avec ou même sans laveur Richmond (3).

Il comporte 5 grandes divisions, comportant chacune 10 subdivisions. Soient 50 petites divisions au total. Chaque grande division correspond à $1 \mathrm{gr}$. de matière grasse par litre de lait. Chaque petite

(1) Nous verrons ci-après qu'il s'agit souvent de lécithine brûlée.

(2) Cet appareil est construit par la maison GERBER : Butyromètre à laits écrémés et caséines. Modèle des "Fermiers Réunis ». Nous montrerons ultérieurement l'usage qu'on doit en faire pour le dosage de la matière grasse des caséines au cours d'une étude sur cette question.

(3) Il est recommandé de rincer ces appareils à l'alcool et à l'éther pour éviter de garder des traces de matière grasse. 
division correspond à 0 gr. 1 par litre. On peut apprécier (bien qủe cela soit inutile) la demi-petite division.

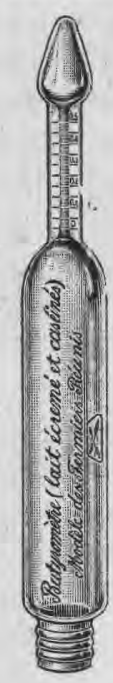

Butyromètre à lait écrémé et caséines modèle des a Fermiers Réunis * Nota. - C'est par erreur que ce cliché porte les graduations $0,1,0,2,0,3$, L'appareil est gradué $1,2,3,4,5$.

Ce tube permet de doser des laits ayant jusqu'à 5 gr. de matière grasse par litre, avec la préeision du décigramme.

2. MOdE OPÉRATOIRE A EMPLOYER. - La méthode Gerber prescrit le mélange de $11 \mathrm{~cm}^{3}$ (ou 22) de lait avec $10 \mathrm{~cm}^{3}$ (ou 20) d'acide sulfurique $\left(\mathrm{D}=1,820\right.$ à 1,825 ) et $1 \mathrm{~cm}^{3}$ (ou 2) d'alcool amylique bouillant à $129^{\circ}-130^{\circ}$. Le caractère essentiel de notre mode opératoire est de ne faire usage que de $20 \mathrm{~cm}^{3}$ de lait (et 20 d'acide Gerber). L'idée qui nous a guidés est l'utilisation de ce même butyromètre à l'analyse des caséines, où, en employant 2 gr. de produit, les mêmes graduations donneront directement, sans calcul, le pourcentage de matière grasse.

Ainsi, une des cinq grandes divisions de notre appareil représente 1 gr. par litre de graisse (1) dans le lait écrémé et $1 \%$ de graisse dans la caséine (voir notre prochain article). D'ailleurs, l'usage de $20 \mathrm{~cm}^{3}$ de lait (au lieu de 22 dans la méthode Gerber classique) ne crée aucune complication. Enfin, il se justifie pour les raisons suivantes :

On sait qu'il est important, dans la méthode Gerber, de ne pas s'écarter, pour l'acide, des densités 1,820-1,825. Quand on utilise du lait entier, la quantité d'eau introduite par le lait est d'environ $840 \mathrm{~cm}^{3}$ par litre. Avec du lait écrémé, la quantité d'eau introduite est de $910 \mathrm{~cm}^{3}$ par litre (puisqu'il n'y a plus de matière grasse). Pour un même volume de lait, en passant du lait entier au lait écrémé, on amène donc plus d'eau dans le butyromètre. Or, la méthode Gerber a été mise au point sur du lait entier.

Nous nous sommes demandé si cet écart modifiait le chiffre de matière grasse trouvé. Voici comment nous avons opéré :

Pour amener dans le butyromètre une quantité d'eau qui corresponde aux conditions exactes du Gerber classique sur laits entiers, il faudrait n'introduire que $22 \times \frac{860}{910}$, ou $20 \mathrm{~cm}^{3} 8$ de lait écrémé. En faisant l'essai à plusieurs reprises et en ramenant le chiffre trouvé au. volume de $22 \mathrm{~cm}^{3}$, on s'est aperȩu qu'on trouvait exactement le même

(1) Autrement dit, en valeur absolue, une grande division contient $20 \mathrm{mgr}$. de matière grasse pure, lue dans les conditions de température du dosage. 
chiffre. L'écart de $1 \mathrm{~cm}^{3} 2$ d'eau (22-20,8) est donc sans influence sur le résultat. En considérant que le volume de 20,8 est celui qui correspond aux conditions classiques, et qu'un écart de $1 \mathrm{~cm}^{3} 2$ sur la quantité d'eau (en plus) était sans importance, nous étions fondés à penser qu'un écart plus faible dans l'autre sens n'aurait pas d'inconvénients.

Retranchons done $0 \mathrm{~cm}^{3} 8$ d'eau; autrement dit, utilisons $20 \mathrm{~cm}^{3} \mathrm{de}$ lait écrémé au lieu de 20,8 , chiffre optimum. L'expérience montre, tous calculs faits, qu'on obtient le même résultat.

En résumé, les trois combinaisons :

22 de lait écrémé pour 20 d'acide Gerber (conditions habituelles des laits entiers),

20,8 de lait écrémé pour 20 d'acide Gerber (conditions optima pour laits écrémés),

20 de lait écrémé pour 20 d'acide Gerber (conditions proposées par nous),

donnent le même résultat (en ramenant par le calcul au même volume).

D'ailleurs, l'expérience nous a montré que, dans la méthode Gerber classique sur laits entiers, on pouvait accroître la densité de l'acide (comme nous le faisons ici en diminuant la quantité de lait, c'est-à-dire d'eau) sans nuire au chiffre de matière grasse. Ainsi, un lait à 40,5 (moyenne de huit essais avec acide Gerber) refait avec de $1^{\prime}$ acide sulfurique pur (à $66^{\circ} B^{e}$ ) donne 41,5 (moyenne de huit essais) ; seulement, la matière grasse est très fortement colorée et ne peut se lire qu'au voisinage d'une lampe électrique. On peut en déduire qu'une faible augmentation de densité du mélange final (par suite de l'addition d'une quantité de lait plus petite) est sans importance sur le résultat. Les concordances rapportées ci-dessus n'ont donc rien de surprenant.

On peut prendre $20 \mathrm{~cm}^{3}$ de lait écrémé pour 20 d'acide Gerber au lieu de $20 \mathrm{~cm}^{3} 8$ (eonditions théoriques) ou $22 \mathrm{~cm}^{3}$ (conditions des laits entiers). On nous excusera d'avoir insisté sur ce point; il était important de le fixer avec certitude, puisqu'il constitue la base de notre mode opératoire. Pour le reste, tenons-nous en d'abord aux prescriptions strictes du Dr GERBER [20], édictées spécialement pour le cas des laits écrémés.

\section{D'où le mode opératoire provisoire :}

Dans notre butyromètre spécial à laits écrémés, on introduira :

$20 \mathrm{~cm}^{3}$ d'acide sulfurique Gerber $(\mathrm{D}=1,825)$;

$20 \mathrm{~cm}^{3}$ de lait écrémé ;

$2 \mathrm{~cm}^{3}$ d'alcool amylique.

Il est indifférent de mettre l'alcool avant ou après le lait.

L'appareil est bouché, retourné plusieurs fois avant d'agiter violemment, puis secoué pendant 3 minutes. 
On le porte ensuite 5 minutes dans un bain-marie à $50^{\circ}$.

On centrifuge 3 minutes à 1.200 tours (1).

On remet au bain-marie à $50^{\circ}$ pendant 5 minutes.

On déplace le bouchon, pour que la matière grasse soit maintenue dans la panse de l'appareil et ne monte pas encore dans la colonne graduée.

On centrifuge une deuxième fois comme précédemment.

Enfin, on porte dans un bain-marie à $65^{\circ}$ pendant 5 minutes, pour faire la lecture.

Remarques. -1 . Pendant le séjour au bain-marie à $65^{\circ}$, on surveillera la montée de la graisse dans l'échelle et on déplacera le bouchon pour éviter que le beurre ne monte dans la petite ampoule supérieure. Il faut chercher à maintenir la graisse dans la limite des graduations, afin d'éviter, au cours de la lecture, d'avoir à déplacer à nouveau le bouchon, ce qui tapisserait de graisse les parois du tube et donnerait un chiffre trop faible. Cette recommandation, facile à suivre, est très importante, car il s'agit ici de très petites quantités de matière grasse. L'opération complète (que l'on peut mener en série) dure moins d'une demi-heure.

2. Le butyromètre que nous a construit la maison GERBER, est bien entendu gradué pour la quantité de $20 \mathrm{~cm}^{3}$ de lait introduite, c'est-à-dire différent de ses autres modèles. Nous en avons vérifié quelques exemplaires en sectionnant l'échelle aux deux extrémités, en' affleurant à 0 avec de la cire à cacheter et en remplissant la colonne de beurre pur, fondu, filtré et sec. En opérant à $65^{\circ}-70^{\circ}$, le poids de matière grasse pure nécessaire à remplir complètement les 50 divisions représente $100 \mathrm{mgr}$. à moins de $\mathrm{I} \mathrm{mgr}$. près (2).

La graduation du tube est donc exacte.

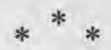

II.

RESULTATS OBTENUS.

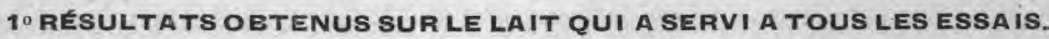

- Le même lait qui a servi précédemment à l'étude des méthodes Adam et Röse-Gottlieb, a été, les mêmes jours, analysé par la méthode de Gerber à l'aide de huit butyromètres du nouveau modèle.

On a trouvé un chiffre moyen de 2,3 par litre, avec des écarts extrêmes ne dépassant pas 0,1 par litre. Refait huit jours plus tard (conservé en pièce chaude), ce lait donne encore 2,3 à l'aide de notre butyromètre.

| L'écart constaté entre les méthodes d'extraction qui ont donné

(1) Il est bon de faire usage dés tubes de euivre grand modèle $(15 \mathrm{~cm}$. de long) pour la centrifugation.

(2) Nous verrons plus loin pourquoi, en introduisant dans le butyromètre ce même poids. de matière grasse et en appliquant la technique de Gerber, on ne retrouve pas lo poids de matière grasse introduite. 
2,7 avait d'abord été mis sur le compte de matières non grasses (caséine, lactose). Les analyses relatées plus haut nous ont fait abandonner cette hypothèse, et ce n'est que lorsque nous eûmes achevé les dosages de lécithine que nous pûmes émettre l'hypothèse que la lécithine n'était pas dosée au Gerber.

\section{2。 ETUDE DU COMPORTEMENT DE LA LECITHINE DANS LE DOSAGE GERBER.}

a) Petites quantités. - Introduisons dans nos butyromètres à lait écrémé $4,10,20,40,80 \mathrm{mgr}$. de lécithine pure. On devrait lire: $2,5,10,20,40$ petites divisions. Or, en opérant comme pour un lait, on lit, respectivement : 0 , traces, 4,13 à 14 et 15 à 30 divisions. On détruirait done, dans le dosage, de 10 à $20 \mathrm{mgr}$. de lécithine.

$\mathrm{Si}$ on remet la lécithine séparée, au contact avec l'acide dans la panse et qu'on centrifuge et lise à nouveau, on s'aperçoit gu'il en est disparu bien davantage (alors qu'on n'a que de faibles différences en procédant ainsi sur du beurre pur). La lécithine se détruit done de plus en plus. D'ailleurs, dès la première centrifugation, elle se présente dans le canal sous forme d'une masse très noire et floconneuse qui ne ressemble en rien à de la matière grasse.

b) Doses importantes. - En faisant le même essai dans un butyromètre à lait entier et en introduisant des quantités plus importantes de lécithine pure (240 mgr. correspondant à environ 22 divisons), on constate qu'une masse complètement noire occupe presque le volume qu'elle devrait occuper. Il manque une ou deux divisions (c'est-à-dire 10 à $20 \mathrm{mgr}$., comme dans l'essai précédent). Mais si on mélange à nouveau, la perte s'accentue beaucoup. De toute façon, on ne saurait fonder un procedé de dosage de la lécithine sur la méthode Gerber, d'abord parce qu'il s'en dissout une partie qui devient incentrifugeable et ensuite parce que le reste est attaqué, brûlé et ne donnerait pas des résultats constants. La quantité détruite (10 à $20 \mathrm{mgr}$, en valeur absolue) correspondrait à 1 ou $2 \mathrm{gr}$. de lécithine par litre de lait, dose supérieure (ou au plus égale) à la teneur en lécithine du lait de vache.

D'après Woon [21], il y aurait dans le lait 0,5 de lécithine. Donc toute la lécithine du lait est dissoute dans l'essai Gerber. Il pourrait arriver, dans certains laits riches en cette substance, qu'une partie échappe à la dissolution et vienne, comme dans les essais relatés cidessus, donner un anneau noir dans la tige graduée du tube. Or, ce phénomène apparaît quelquefois et pourrait s'expliquer de cette façon.

c) Mélanges de lécithine et matière grasse pure. - Il était intéressant, enfin, d'introduire dans notre butyromètre de la matière grasse pure et de la lécithine pour constater la disparition de celle-ci 
et le maintien de celle-là et voir ce qui se passe quand il y a beaucoup de lécithine.

On a introduit une dose de beurre pur devant occuper 26 divisions et une dose de lécithine devant occuper 24 divisions. Après le dosage dans les conditions normales, on a lu 26 divisions de matière grasse pure limpide, d'aspect normal et, entre celle-ci et la couche acide sousjacente, un eylindre noir opaque occupant 11 divisions, puis 7 après une nouvelle centrifugation. On a obtenu le même résultat en utilisant dans le butyromètre (avec l'acide) de l'eau ou bien un lait artificiel synthétique. On a également observé le même résultat en faisant le mélange préalable beurre et lécithine, ou bien en introduisant la lécithine dans un tube contenant un essai de beurre pur déjà terminé. En aucun cas, la lécithine ne se mélange à la matière grasse pure. Sa présence ne gêne en rien la lecture du beurre pur. Elle se place (quand il y en a assez pour qu'elle apparaisse) entre le niveau inférieur de la couche de graisse et le niveau supérieur de la solution acide du tube. Enfin, il arrive quelguefois (en présence d'un petit excès de lécithine) que celle-ci n'apparaisse qu'à la seconde centrifugation et soit invisible à la première.

En Résumé. - Aux doses où elle existe dans le lait, la lécithine n'apparaît pour ainsi dire jamais. Et quand il s'en trouve un peu plus, son apparition n'est nullement gênante. Elle n'est, en tout cas, jamais comptée dans la lecture de matière grasse.

La méthode Gerber donne des, chiffres de matière grasse exempts de lécithine.

3० COMPARAISON DES RÉSULTATS DES MÉTHODES D'EXTRACTION ET GERBER. - Nous sommes donc en présence des résultats suivants : Matière grasse pure exempte de lécithine (dosée au Gerber) : 2,3. Chiffres moyens des méthodes d'extraction : 2,74 à 2,77 d'extrait éthéré total.

Ces chiffres se décomposent en:

Lécithine : 0,21 à 0,22 ;

Impuretés autres que la lécithine : 0,09 à 0,10 ;

Matière grasse supposée pure (Adam-Gottlieb) : 2,43 à 2,44.

Il subsiste donc entre ces derniers chiffres et ceux du Gerber un écart de 0,1 à 0,15 , qui, normalement, aurait dû être nul.

On peut évidemment le négliger. Mais nous avons acquis la conviction, aux cours des essais sévères que nous avons poursuivis, que cet écart n'est pas dû aux incertitudes d'analyses. D'ailleurs, dans d'autres essais sur d'autres laits, nous avons eu quelquefois des écarts plus importants.

Nous montrerons, dans la suite de ce travail, que c'est le mode opératoire de Gerber qui est en cause et que l'utilisation rationnelle des butyromètres à lait écrémé (le nôtre en particulier) exige une 
technique un peu différente. La technique de Gerber n'arrive pas (en deux courtes centrifugations) à extraire toute la matière grasse d'un lait écrémé.

Quoi qu'il en soit, la comparaison de ces chiffres montre que, sur le lait écrémé, les méthodes Adam et Röse-Gottlieb (bien conduites) donnent des chiffres identiques entre eux, un peu différents de ceux de la méthode Gerber (ici, 0,47 d'écart maximum par litre de lait). Cet écart est causé, d'une part, par la présence dans l'extrait éthéré de lécithine et $d$ 'une petite quantité de matières étrangères (lactose, caséine), d'autre part, par un léger déficit de la méthode Gerber dans sa technique classique.

Remarque. - A titre de documentation, rappelons que certains auteurs ont depuis longtemps vérifié l'exactitude du procédé Gerber.

Mais, lorsque Dornic [22], par exemple, compare les chiffres du Gerber à ceux obtenus par épuisement à l'éther de l'extrait sec, il conclut à une exactitude suffisante du Gerber, parce que les écarts entre les deux procédés n'excèdent pas $1 \mathrm{gr}$. par litre (aussi bien sur des laits écrémés que sur des laits entiers). Il trouve ainsi : 4 gr. 2, 2 gr., traces, au Gerber, au lieu de : 5,5, 3,8, 1,7, à la reprise éthérée de l'extrait. Il est évidemment nécessaire d'être plus sévère, même pour une méthode rapide et pratique comme le Gerber.

Mais nous venons de voir que l'écart entre les deux séries de méthodes est en réalité beaucoup plus faible si l'on applique correctement. les méthodes d'Adam ou de Gottlieb. Nous verrons même, dans la suite de cette étude, que cet écart peut être rendu à peu près nul si l'on a soin de suivre, pour la méthode Gerber, une technique améliorée, qui donnera toute la matière grasse.

Il n'y aura plus, en définitive, entre ces méthodes que l'écart négligeable $(0,2$ par litre, ou même moins) dû aux traces d'impuretés. dosées dans l'Adam et le Röse-Gottlieb.

\section{TROISTEME PARTIE.}

\section{RÉSUMÉ.}

1. Les méthodes Adam et Röse-Gottlieb donnent des résultats identiques sur le lait écrémé et très constants, sous réserve d'appliquer à la lettre le mode opératoire défini par ADAM et rappelé dans cette étude.

2. Les extraits éthérés obtenus contiennent environ 0 gr. 1 (par litre de lait) de matières étrangères non grasses (lactose, caséine, etc.), surcharge pratiquement négligeable.

3. Ils contiennent en outre une petite quantité de lécithine (0,2 par litre de lait), qui paraît assez voisine de la lécithine totale du lait écrémé soumis à nos essais.

4. Si on considère la lécithine comme devant faire partie de la 
matière grasse normale du lait, ces deux méthodes donnent des chiffres pratiquement exacts et identiques.

5. La méthode Gerber peut se prêter au dosage exact de la matière grasse du lait écrémé, sous réserve de faire usage d'un butyromètre remplissant un certain nombre de conditions pratiques, qui sont réalisées dans le butyromètre spécial que nous avons créé.

6. La méthode Gerber ne dose pas les lécithines.

Le volume de matière grasse pure lu au Gerber (lait entier ou écrémé) n'est pas affecté par la présence des quantités quelconques de lécithines. Le chiffre obtenu sur le lait écrémé est légèrement inférieur au chiffre théorique.

7. L'écart total entre les méthodes par extraction (correctement utilisées) et la méthode Gerber comprend, dans nos essais, en dehors de 0,2 de lécithine et 0,1 de matières étrangères contenues dans l'extrait, un indéterminé négligeable de 0,1 à 0,15 par litre de lait (quelquefois plus sur d'autre laits) qui provient d'un déficit dans la technique Gerber classique, ainsi que nous le montrerons.

\section{If. CONCLUSIONS.}

1. Les méthodes Adam et Röse-Gottlieb donnent toute la matière grasse du lait écrémé, y compris une grande partie (et peut-être la totalité) des lécithines. Si on considère la lécithine comme devant faire partie de la matière grasse normale du lait, ces méthodes sont pratiquement exactes et identiques entre elles.

2. La méthode Gerber donne la matière grasse pure exempte de lécithine. Elle peut se prêter à des dosages exacts. Nous avons créé, à cet effet, un butyromètre permettant le dosage facile de la matière grasse des laits écrémés.

3c L'écart qui subsiste entre les méthodes par extraction et la méthode Gerber est dû à une déficience de celle-ci, ainsi que nous le montrerons en indiquant le mode opératoire qu'il convient d'adopter pour y parer.

\section{BIBLIOGRAPHIE.}

[1] Ch. Brroux. Des méthodes chimiques d'appréciation de la matière grasse et de l'extrait sec dans le lait. Congrès de l'aliment, du bétail bovin et du Contrôle laitier (Octobre 1925).

[2] OrLa-Jensen. Le procédé Höyberg pour la détermination de la matière grasse dans le lait et la crème. Le Lait, mars 1923, 177.

[3] A. Adam. Thèse de la Faculté de Médecine de Paris, 1879.

[4] A. Adam. Nouvelle méthode d'analyse du lait. Annales d'Hygiène et de Médecine légale, $1879,3^{\mathrm{e}}$ série, 1.

[5] A. Adam. Nouveau procédé pour l'analyse complète du lait. Répertoire de Pharmacie, $1879,7,150$.

[6] Marchand. Bulletin de la Société Chimique, 29, 147.

[7] Magnier de da Source. Répertoire de Pharmacie, 1879, 7, 303. 
[8] A. AdaM. Journal de Pharmacie et de Chimie, 1881.

[9] KrrNG. Méthodes actuelles d'expertises employées au Laboratoire municipal de Paris. 1921; Dunod, éd.

[10] Hinard. Analyse des laits (Encyclopédie scientifique des aide-mémoire). Gauthier-Villars, éd.

[11] BRuno Röse. Journal d'Industrie laitière, 1888, 157.

[12] Methlł̀re. Journal de Pharmacie et de Chimie, 15 février 1894.

[13] Mernlìne. Journal de Pharmacie et de Chimie, 16 juin 1904.

[14] Metrlère. Bulletin des Sciences pharmacologiques, mai 1908.

[15] Ch. Brioux. Annales de Chimie analytique, 15 octobre 1911, 373.

[16] Bordas et StG. de Raczkowski. C. R. Acad, des Sc., 1902, 134, 1592.

[17] Ch. Marie. C. R. Acad. des Sc, 1899 (2e semestre), 766.

[18] Gutkrn, Pflügers Arch. f. d. ges. Physiol., 95, 107.

[19] Chapman. Journal of Dairy Science, novembre 1928, 11, 6, 429.

[20] Dr N. Gerker et Dr A. Otriker. Traité pratique des essais du lait et produits de laiterie. $8^{\mathrm{e}}$ édition, 1919 ; K. J. Wyss-Erben, éd., Berne.

[21] Wood. The Journal of Biological Chemistry, 1905.

[22] DoRnIc. Journal d'Industrie laitière, 1896, 74.

\title{
CONSIDÉRATIONS PHYSICO-CHIMIQUES SUR LE FROMAGE (1)
}

\author{
par G. KOESTLER
}

Travail de l'Institut de Laiterie et de Microbiologie du Liebefeld.

Directeur-Professeur : $\mathrm{D}^{\mathrm{r}} \mathrm{R}$. BURRI.

Les progrès de nos connaissances sur la nature des gelées nous permettent de plus en plus de comprendre et d'étudier le fromage considéré en tant que système physico-chimique. Le grand bénéfice qui se dégage de ce point de vue pour la pratique provient de ce que de nombreux processus de transformation du fromage sont étroitement liés à cette nature physico-chimique.

Les recherches futures devront s'appuyer sur ces considérations pour étudier la nature physico-chimique du fromage considéré comme milieu microbien et enzymatique des processus de transformation. Logiquement, on devrait arriver par ce moyen à expliquer toute une série de faits concernant le développement des défectuosités du fromage. Le présent travail est une contribution dans cette direction.

On établira tout d'abord quelques données sur la nature physicochimique de la masse du fromage. Dans la deuxième partie, on étudiera comment les recherches physico-chimiques peuvent aider pratiquement à mieux résoudre des problèmes importants. Dans la dernière partie, on exposera quelques-unes des méthodes de recherches qui permettront d'établir certaines propriétés physiques du fromage.

(1) Travail paru dans Landwirtschaftliches Jahrbuch der Schweiz, 1931, p. 421. 